\title{
Invariance of quantum optimal control fields to experimental parameters
}

\author{
Emanuel F. de Lima* \\ Instituto de Geociências e Ciências Exatas, UNESP-Univ Estadual Paulista, Rio Claro, São Paulo 13506-900, Brazil
}

\author{
Tak-San $\mathrm{Ho}^{\dagger}$ and Herschel Rabitz \\ Department of Chemistry, Frick Laboratory, Princeton University, Princeton, New Jersey 08544, USA
}

(Received 27 March 2014; published 21 April 2014)

\begin{abstract}
We consider an ensemble of closed finite-level systems described by a density matrix where the goal is to find an optimal control field to maximize the expectation value of an observable. The eigenvalues of the initial density matrix are assumed to depend on an experimental parameter (e.g., the temperature), whereas the eigenvalues of the observable may depend on an additional application-specific experimental parameter. We show that an optimal control will remain optimal for all such experimental parameters, if the relative ordering, by magnitude, of the eigenvalues of the initial density matrix as well as of the observable is unaltered regardless of the parameter values. More generally, we show like invariance of a control associated with any particular critical point on the corresponding control landscape. The invariance of control laser fields with respect to temperature is illustrated for vibrational excitation of diatomic molecules and photoassociation of atoms.
\end{abstract}

DOI: 10.1103/PhysRevA.89.043421

PACS number(s): $32.80 . \mathrm{Qk}, 42.50 . \mathrm{Hz}, 33.80 .-\mathrm{b}, 02.60 . \mathrm{Pn}$

\section{INTRODUCTION}

Quantum control is generally concerned with finding an optimal field that can steer a system from an initial state to a final state that maximizes the expectation value of an observable [1-10]. In the laboratory, the initial state and the observable often depend on continuously adjustable experimental parameters. Naturally, the outcome of applying an optimal control will produce a final expectation value that depends on these experimental parameters. Here we seek to establish conditions for when a control field remains optimal, regardless of the values of the parameters, such that an optimal field determined at one set of parameter values will again produce an optimal outcome at any other values of the parameters. An understanding of the physical conditions consistent with such invariance properties for the optimal control field is important in the laboratory as well as for performing control designs.

The energy spectrum of a molecule is composed of both discrete bound rotational-vibrational-electronic energy levels and continuous ionization or dissociative channels. In practice, however, many situations can be viewed as a finite $N$-level quantum system, including possibly by proper discretization of continuum states. In addition, some systems (e.g., spins) are inherently finite dimensional. Thus, in this work the corresponding field-free Hamiltonian $H_{0}$, dipole moment operator $\mu$, density operator $\rho(t)$, and observable $O$ are all assumed to be effectively represented by $N$-dimensional Hermitian matrices. As a result, the initial state $\rho(0)$ can be written in diagonal form

$$
\rho(0)=\sum_{k=1}^{N} p_{k}\left|\phi_{k}\right\rangle\left\langle\phi_{k}\right|,
$$

\footnotetext{
*eflima@rc.unesp.br

†tsho@princeton.edu

${ }^{\ddagger}$ hrabitz@princeton.edu
}

where the eigenvalues $\left\{p_{k}\right\}$ specify the probabilities (statistical weights) that the system initially resides in the various orthogonal pure states $\left\{\left|\phi_{k}\right\rangle\right\}$. Correspondingly, the observable $O$, characterized by a set of eigenvalues $\left\{\lambda_{j}\right\}$, can be written as

$$
O=\sum_{j=1}^{N} \lambda_{j}\left|\psi_{j}\right\rangle\left\langle\psi_{j}\right|
$$

with $\left\{\left|\psi_{j}\right\rangle\right\}$ being the associated eigenstates of $O$.

The optimal control problem for a closed system may be formulated as maximization of the cost functional $J[\varepsilon]=$ $\operatorname{Tr}\left[\rho\left(t_{f}\right) O\right]$, i.e.,

$$
\max _{\varepsilon(\cdot)} J[\varepsilon],
$$

where $J[\varepsilon]$ is the expectation value of $O$ at final time $t_{f}$ and forms a control landscape as a functional of the squaredintegrable control $\boldsymbol{\varepsilon}(t) \in \mathcal{L}^{2}\left[0, t_{f}\right]$ [11]. The key features of the control landscape $J[\varepsilon]$ are the critical points where $\delta J / \delta \boldsymbol{\varepsilon}(t)=0, t \in\left[0, t_{f}\right]$. Without loss of generality, consider that the eigenvalues of both the initial density operator and the target operator are arranged in the decreasing order $p_{1} \geqslant p_{2} \geqslant$ $\cdots \geqslant p_{k} \geqslant \cdots \geqslant p_{N}$ and $\lambda_{1} \geqslant \lambda_{2} \geqslant \cdots \geqslant \lambda_{j} \geqslant \cdots \geqslant \lambda_{N}$. Assuming that (1) the system is fully controllable [12-14], (2) the control to the final state differential map $\delta \varepsilon(\cdot) \mapsto \delta \rho\left(t_{f}\right)$ is surjective [15], and (3) that the controls are unconstrained $[16,17]$, then each critical point corresponds to a permutation of the eigenvalues of $\rho(0)$ or $O$ such that [18-25]

$$
J_{\text {critical }}[\varepsilon]=\sum_{i=1}^{N} p_{i} \lambda_{\pi(i)}=\sum_{i=1}^{N} p_{\pi^{-1}(i)} \lambda_{i},
$$

where $\pi(i)$ denotes a permutation of $N$ indices and $\pi^{-1}(i)$ its inverse. In particular, the upper limit for the expectation value of the observable is

$$
J_{\max }[\boldsymbol{\varepsilon}]=\sum_{i=1}^{N} p_{i} \lambda_{i}
$$


which is the maximum value attainable by an optimal control field.

The weights $p_{k}$ of the initial density matrix $\rho(0)$ will often depend on some adjustable experimental parameter $X$, i.e., $p_{k}=p_{k}(X)$. For example, the statistical weights (i.e., the Boltzmann factors) for a thermal ensemble are determined by the temperature $X \equiv T$. The observable may be chosen a priori such that its eigenvalues $\lambda_{j}$ are arbitrary functions of some parameter $Y$, i.e., $\lambda_{j}=\lambda_{j}(Y)$ in a suitably designed measurement apparatus. As a result, the value of $J_{\text {critical }}[\varepsilon]$ at a critical point of the landscape, including the upper limit for the optimal solution, will generally depend on the experimental parameters $X$ and $Y$. However, this paper aims to present sufficient conditions whereby a control field $\boldsymbol{\varepsilon}$ corresponding to a particular critical point $J_{\text {critical }}[\varepsilon]$ for one value of $X$ and $Y$ also corresponds to the same critical point for other values of these parameters. The special case of interest is an optimal control remaining optimal to produce $J_{\max }[\varepsilon]$, regardless of the values for $X$ or $Y$. The key to these invariance prospects lies in the fact that the field serves to create a unitary permutation matrix from the dynamics thereby providing a particular critical point outcome $J_{\text {critical }}[\varepsilon]$ [18-25].

The paper is organized as follows. Section II presents sufficient physical conditions for the existence of critical point preserving fields. The symmetric roles played by $\rho(0)$ and $O$ in the expectation value $J$ are emphasized in the formulation. Section III presents illustrations for the molecular vibrational excitation and for the photoassociation in a thermal gas of atoms; in both cases the adjustable laboratory parameter is the temperature $X=T$. Concluding remarks are given in Sec. IV. Appendix A introduces a monotonically convergent iteration scheme to find optimal control fields by extending the TBQCP method to the density matrix framework [26]. Appendix B describes a search scheme to find saddle critical points on the control landscape.

\section{CRITICAL POINT INVARIANCE}

Consider an ensemble of $N$-level quantum systems with the initial state specified by a density matrix $\rho(0, X)$,

$$
\rho(0, X)=\sum_{k=1}^{N} p_{k}(X)\left|\phi_{k}\right\rangle\left\langle\phi_{k}\right|,
$$

where the set of eigenstates $\left\{\left|\phi_{k}\right\rangle\right\}$ of $\rho(0, X)$ forms an orthornormal basis and the initial population distribution $\left\{p_{k}(X)\right\}$ over $\left\{\left|\phi_{k}\right\rangle\right\}$ is determined by the values of some laboratory parameter $X$. Moreover, we assume that all $N$ eigenstates $\left|\phi_{k}\right\rangle$ are completely discernible experimentally regardless of whether they are degenerate or not. For example, states with degeneracy in the magnetic quantum number $m$ can in principle be distinguished in the laboratory using appropriate polarized or DC fields. Thus, even upon the occurrence of degenerate eigenvalues $p_{k}(X)$, their association with a particular state $\left|\phi_{k}\right\rangle$ can be established in the laboratory. Similarly, the observable matrix is assumed to depend on an application-specific experimental parameter $Y$,

$$
O(Y)=\sum_{j=1}^{N} \lambda_{j}(Y)\left|\psi_{j}\right\rangle\left\langle\psi_{j}\right|
$$

with $\left\{\left|\psi_{j}\right\rangle\right\}$ being eigenstates of $O(Y)$. The matrix $O(Y)$ in the $\left\{\left|\phi_{k}\right\rangle\right\}$ basis can be obtained by the unitary transformation $D$,

$$
O(Y)=D^{\dagger} \Lambda(Y) D,
$$

where an element $(O)_{k l}$ of the observable matrix in Eq. (4) is $\left\langle\phi_{k}|O| \phi_{l}\right\rangle$, with $D_{j k}=\left\langle\psi_{j} \mid \phi_{k}\right\rangle$, and $\Lambda(Y)$ is a diagonal matrix composed of the eigenvalues $\lambda_{1}(Y), \ldots, \lambda_{N}(Y)$. In practice, the $Y$ dependence of $O(Y)$ may be constructed with considerable freedom, assuming that the projection into the eigenstate $\left|\psi_{j}\right\rangle$ can be observed in separate, identically setup experiments. Then, the outcomes can be weighted at will with eigenvalues $\left\{\lambda_{j}(Y)\right\}$. We point out the special structure assumed for the initial state $\rho(0, X)$ and for the observable $O(Y)$, expressed in Eqs. (2) and (3): while their eigenvalues depend on the parameters $X$ and $Y$, the corresponding eigenstates do not depend on these parameters.

The cost functional associated with the control problem of Eq. (1) can be written as

$$
J[X, Y, \varepsilon] \equiv \operatorname{Tr}\left\{\rho\left(t_{f}, X\right) O(Y)\right\}=\operatorname{Tr}\left\{\rho(0, X) O\left(t_{f}, Y\right)\right\},
$$

where

$$
\rho\left(t_{f}, X\right)=U\left(t_{f}, 0\right) \rho(0, X) U^{\dagger}\left(t_{f}, 0\right)
$$

and

$$
O\left(t_{f}, Y\right)=U^{\dagger}\left(t_{f}, 0\right) O(Y) U\left(t_{f}, 0\right)
$$

The propagator $U\left(t_{f}, 0\right)$ dictates the evolution of the quantum system from $t=0$ to $t=t_{f}$ in the presence of the control field $\boldsymbol{\varepsilon}(t)$. The roles played by $\rho(0, X)$ and $O(Y)$ are physically distinct, but mathematically interchangeable in the optimal control formulation, manifested in Eqs. (5) to (7). The control problem may be formulated as either steering forward from $\rho(0, X)$ to $\rho\left(t_{f}, X\right)$ to maximize the expectation value $\operatorname{Tr}\left\{\rho\left(t_{f}, X\right) O(Y)\right\}$ or steering backward from $O(Y)$ to $O\left(t_{f}, Y\right)$ to maximize the equivalent expectation value $\operatorname{Tr}\left\{\rho(0, X) O\left(t_{f}, Y\right)\right\}$. Substituting Eqs. (2) and (4) in (5), we obtain

$$
\begin{aligned}
J[X, Y, \boldsymbol{\varepsilon}] & =\operatorname{Tr}\left\{\rho(0, X) \Theta^{\dagger} \Lambda(Y) \Theta\right\} \\
& =\sum_{k=1}^{N} p_{k}(X)\left\langle\phi_{k}\left|\Theta^{\dagger} \Lambda(Y) \Theta\right| \phi_{k}\right\rangle \\
& =\sum_{k=1}^{N} p_{k}(X) \sum_{j=1}^{N} \lambda_{j}(Y)\left\langle\phi_{k}\left|\Theta^{\dagger}\right| \psi_{j}\right\rangle\left\langle\psi_{j}|\Theta| \phi_{k}\right\rangle,
\end{aligned}
$$

where

$$
\Theta \equiv D U\left(t_{f}, 0\right)
$$

is a unitary matrix and $U\left(t_{f}, 0\right)$ is understood to be in the $\left\{\left|\phi_{k}\right\rangle\right\}$ basis.

The so-called kinematic critical points of the control landscape are associated with particular unitary transformations of $\rho(0, X)$ and $O(Y)$, according to Eqs. (6) and (7), such that $\rho\left(t_{f}, X\right)$ and $O(Y)$ commute [25], i.e.,

$$
\left[\rho_{\text {critical }}\left(t_{f}, X\right), O(Y)\right]=0,
$$

or, equivalently, $\rho(0, X)$ and $O\left(t_{f}, Y\right)$ commute, i.e.,

$$
\left[\rho(0, X), O_{\text {critical }}\left(t_{f}, Y\right)\right]=0 .
$$


The kinematic critical points correspond to either global extrema (maxima or minima) or to local saddles of the control landscape,

$$
J_{\text {critical }}[X, Y]=\sum_{j=1}^{N} p_{j}(X) \lambda_{\pi(j)}(Y)=\sum_{j=1}^{N} p_{\pi^{-1}(j)}(X) \lambda_{j}(Y)
$$

where

$$
\pi(j)=\{j \rightarrow \pi(j), j=1,2, \ldots, N\}
$$

denotes any one of the permutations of $N$ indices $\{1,2, \ldots, N\}$. Comparing Eqs. (8) and (12), it can be inferred that the evolution operator which leads to a critical point must satisfy the relation

$$
\Theta_{\text {critical }}=D U_{\text {critical }}\left(t_{f}, 0\right)=\Pi,
$$

where $\Pi$ is a permutation matrix. The effect of $\Pi$ is to transform the diagonal matrix $\Lambda(Y)$ into another diagonal matrix $\Pi^{T} \Lambda(Y) \Pi$ or, equivalently, $\rho(0, X)$ into $\Pi \rho(0, X) \Pi^{T}$, rearranging the diagonal elements in a different order.

If the eigenvalues of both $\rho(0, X)$ and $O(Y)$ remain in decreasing order, regardless of the values for the parameters $X$ and $Y$,

$$
p_{1}(X) \geqslant p_{2}(X) \geqslant \cdots \geqslant p_{k}(X) \geqslant \cdots \geqslant p_{N}(X) \geqslant 0
$$

and

$$
\lambda_{1}(Y) \geqslant \lambda_{2}(Y) \geqslant \cdots \geqslant \lambda_{j}(Y) \geqslant \cdots \geqslant \lambda_{N}(Y) \geqslant 0,
$$

then this criterion presents a sufficient condition that $\boldsymbol{\varepsilon}(t)$ will produce the same critical point independent of the values for $X$ and $Y$. The conclusion follows since the ordering of the eigenvalues of $\rho(0, X)$ and $O(Y)$ is independent of $X$ and $Y$, implying that the permutation matrix leading to a particular critical point has the same invariance to $X$ and $Y$. In particular, when $\Pi=\mathrm{I}_{N}$, we obtain the upper limit (i.e., the maximum value attainable by a control field) for the expectation value of the observable,

$$
J_{\max }[X, Y]=\sum_{i=1}^{N} p_{i}(X) \lambda_{i}(Y)
$$

Thus, in general a field $\boldsymbol{\varepsilon}(t)$ that leads to a particular critical point specified by $\Pi$ will still produce the same critical point independent of the experimental parameters $X$ and $Y$. Although the eigenvalue orderings are preserved by assumption, the individual eigenvalues $p_{i}(X)$ and $\lambda_{j}(Y)$ can vary with $X$ and $Y$, implying that the critical value $J_{\text {critical }}[X, Y]$ can depend on $X$ and $Y$. Nevertheless, we have that the achieved critical value $J[X, Y, \boldsymbol{\varepsilon}]$ will satisfy $J[X, Y, \boldsymbol{\varepsilon}] / J_{\text {critical }}[X, Y]=1$ for a control field $\varepsilon(t)$ associated with a particular critical point, independent of $X$ and $Y$.

\section{ILLUSTRATIONS}

As examples of critical point control field invariance to experimental parameters, we consider one-dimensional molecular control problems with the Hamiltonian

$$
H(t)=H_{0}-\mu(r) \varepsilon(t)
$$

where $H_{0}=-\frac{\hbar^{2}}{2 M} \frac{\partial^{2}}{\partial r^{2}}+V(r)$ is the unperturbed molecular Hamiltonian, with reduced mass $M$ and the internuclear potential $V(r)$. Here $\mu(r)$ is the dipole moment operator and $\boldsymbol{\varepsilon}(t)$ is the control electric field, with the dynamics starting at $t=0$ and ending at $t=t_{f}$. An efficient technique to optimize the control field within the density matrix framework is provided in Appendix A. Appendix B presents a method to find saddle points on the control landscape. Both examples will illustrate the basic principles of Sec. II with the temperature being the laboratory adjustable parameter, $X=T$. The dependence of $O$ on $Y$ is put aside here, but that may be included at will (provided that the eigenvalues of $O$ remain monotonic regardless of the value of $Y$ ) without changing the conclusions.

\section{A. Vibrational excitation}

Consider laser-induced vibrational excitation of IBr oriented in a lattice at temperature $T$ with the field coincident with the molecular axis. We assume that no significant relaxation occurs with the lattice during the fast control pulse. A Morse oscillator model is employed for the ground electronic state, $V(r)=D_{e}\left[e^{-\alpha\left(r-r_{e}\right)}-1\right]^{2}$, with parameters in atomic units given by $D_{e}=0.067, r_{e}=4.67, \alpha=0.996$, and $M=89379$ [27]. An analytical form for the dipole function $\mu(r)$ is taken from Ref. [28]. The molecule is modeled with $N=5$ bound vibrational energy levels $E_{v}$ corresponding to the nondegenerate states $\left|\phi_{\nu}\right\rangle, v=1, \ldots, 5$. The energy levels are given by $E_{v}=D_{e}-\hbar^{2} \alpha^{2}(\zeta-v+1)^{2} /(2 M)$, where $\zeta=\sqrt{2 M D_{e}} /(\hbar \alpha)-1 / 2$, implying that $E_{5}>E_{4}>E_{3}>$ $E_{2}>E_{1}$.

The initial density operator is

$$
\rho(0, T)=\frac{1}{Z} \sum_{\nu=1}^{N=5} e^{-\beta E_{\nu}}\left|\phi_{\nu}\right\rangle\left\langle\phi_{\nu}\right|,
$$

where $Z$ is the partition function, $\beta=1 /\left(k_{B} T\right)$, with $k_{B}$ being the Boltzmann constant. The objective is to increase the vibrational energy of the system, corresponding to the operator

$$
O=\sum_{\nu=1}^{N=5} \lambda_{\nu}\left|\psi_{\nu}\right\rangle\left\langle\psi_{\nu}\right|
$$

where $\lambda_{\nu}=E_{N-v+1}$ and $\left|\psi_{\nu}\right\rangle=\left|\phi_{N-v+1}\right\rangle$. The control problem is specified by maximization of the cost functional

$$
J[T, \boldsymbol{\varepsilon}]=\frac{1}{Z} \sum_{\nu=1}^{N=5} e^{-\beta E_{v}} \sum_{v^{\prime}=1}^{N=5} \lambda_{\nu^{\prime}}\left|\left\langle\psi_{\nu^{\prime}}\left|U\left(t_{f}, 0\right)\right| \phi_{\nu}\right\rangle\right|^{2},
$$

at $t_{f}=2$ ps. The monotonically increasing energy levels $E_{v}$ assure the ordering in Eq. (14) and (15) independent of $T$.

From Eq. (16), the maximum value of $J[T, \varepsilon]$ is

$$
J_{\max }[T]=\frac{1}{Z} \sum_{\nu=1}^{N=5} e^{-\beta E_{v}} \lambda_{v}=\frac{1}{Z} \sum_{\nu=1}^{N=5} e^{-\beta E_{\nu}} E_{N-v+1} .
$$

Using the technique of Appendix A and fixing the temperature at $T=500 \mathrm{~K}$, we found a nearly optimal field $\varepsilon^{*}(t)$ starting from a trial field of the form $\varepsilon_{\text {trial }}=\varepsilon_{0} S(t) \sum_{i, j} \sin \left(\omega_{i, j} t\right)$, where $\omega_{i, j}=\left|E_{i}-E_{j}\right| / \hbar \quad(i, j=1, \ldots, N)$ and $S(t)=$ $\sin ^{2}\left(\pi t / t_{f}\right)$ is an envelope function. The resultant optimized field achieved the yield $J\left[T, \varepsilon^{*}\right] / J_{\max }(T) \approx 0.99$ for 

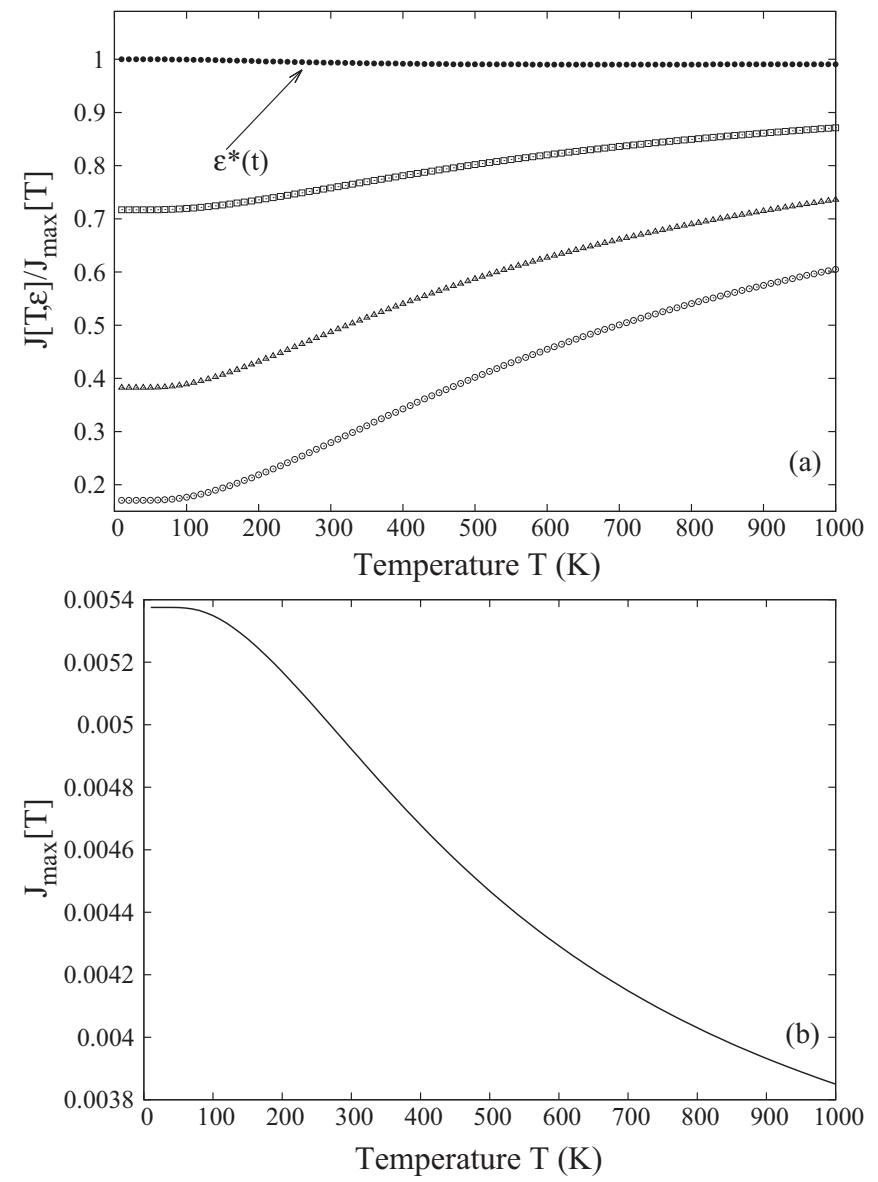

FIG. 1. (a) Ratio $J[T, \varepsilon] / J_{\max }[T]$ as a function of the temperature. Each curve was constructed for a different control field. The curve with filled circles corresponds to the nearly optimal control field $\varepsilon^{*}(t)$ determined at $T=500 \mathrm{~K}$, and then utilized again at all other temperatures $T$. The other curves in (a) with nonoptimal fields do not exhibit temperature invariance. The near invariance to $T$ for the curve created with $\boldsymbol{\varepsilon}^{*}(t)$ occurs, despite the fact that the value of $J_{\max }[T]$ in (b) varies significantly as a function of $T$.

$T=500 \mathrm{~K}$. Figure 1 (a) shows that the ratio $J\left[T, \varepsilon^{*}\right] / J_{\max }[T]$ remains close to 0.99 for all temperatures upon using the nearly optimal field $\boldsymbol{\varepsilon}^{*}$ determined at $500 \mathrm{~K}$ (filled circles), consistent with the analysis of Sec. II. For comparison, Fig. 1(a) also shows the ratio $J[T, \varepsilon] / J_{\max }[T]$ for three other nonoptimal control fields (open circles, triangles, and squares), which were obtained while performing the optimization at $T=500 \mathrm{~K}$. In contrast to $\varepsilon^{*}$, for the three nonoptimal control fields, $J[T, \varepsilon] / J_{\max }[T]$ changes considerably as the temperature is varied. While $J\left[T, \varepsilon^{*}\right] / J_{\max }[T]$ remains near 0.99 for all temperatures in Figs. 1(a), 1(b) shows that $J_{\max }(T)$ given in Eq. (21) decreases significantly with the temperature.

Additionally, consider the following saddle point of the control landscape:

$$
J_{\text {saddle }}[T]=\frac{1}{Z}\left(e^{-\beta E_{1}} \lambda_{2}+e^{-\beta E_{2}} \lambda_{1}\right)+\frac{1}{Z} \sum_{v=3}^{N=5} e^{-\beta E_{v}} \lambda_{\nu} .
$$

We have applied the technique described in Appendix B to find a control field $\boldsymbol{\varepsilon}^{\mathrm{s}}(t)$ producing an outcome close to the intended saddle point, such that $J\left[T, \varepsilon^{\mathrm{s}}\right] / J_{\text {saddle }}(T) \approx 0.98$
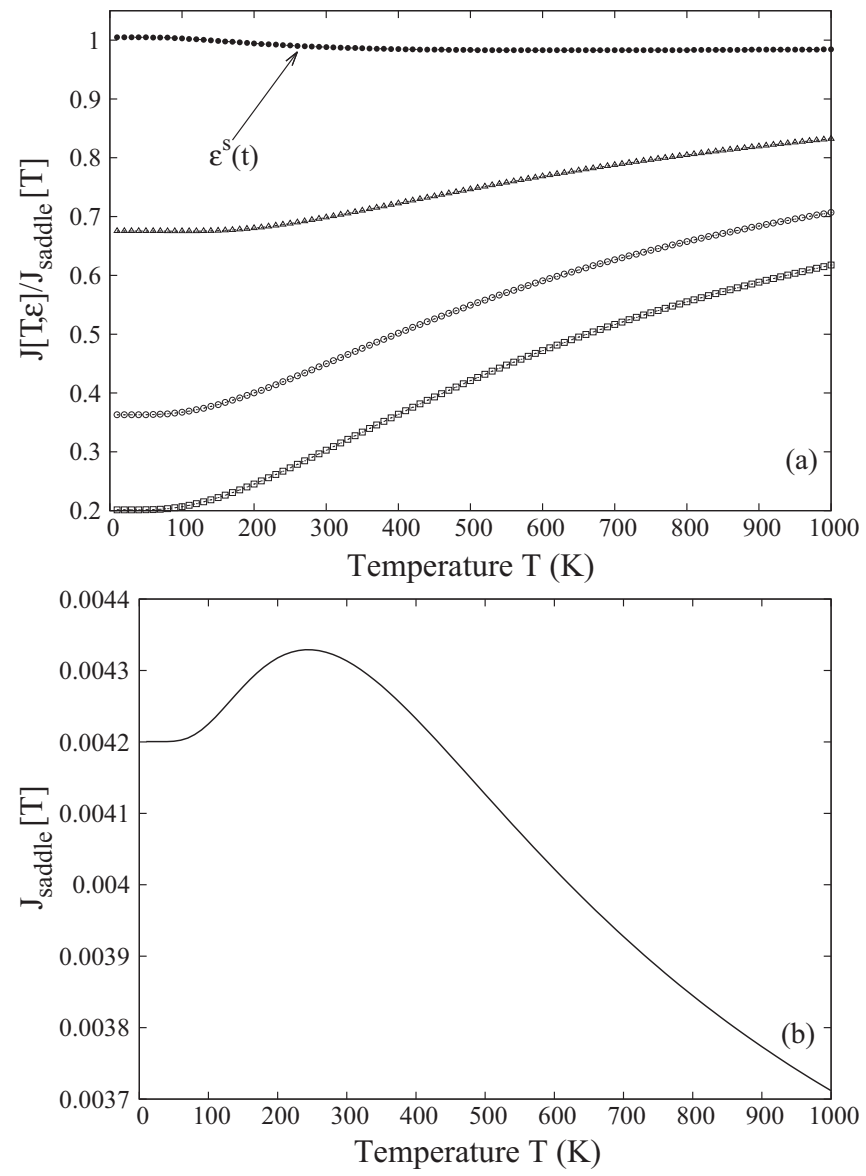

FIG. 2. (a) Ratio $J[T, \varepsilon] / J_{\text {saddle }}(T)$ as a function of the temperature. Each curve was constructed for a different control field identified at $500 \mathrm{~K}$ and then utilized at other temperatures. The curve with filled circles corresponds to the control field $\boldsymbol{\varepsilon}^{s}(t)$ closest to the saddle. The nearly constant value for $J\left[T, \varepsilon^{\mathrm{s}}\right] / J_{\text {saddle }}(T)$ with respect to temperature occurs, despite the saddle point value $J_{\text {saddle }}[T]$ in (b) having a significant nonmonotonic variation with temperature $T$. In contrast, the other curves in (a) obtained with nonsaddle creating fields do not show temperature invariance.

at $T=500 \mathrm{~K}$. Figure 2 (a) shows the ratio $J[T, \varepsilon] / J_{\text {saddle }}[T]$ as a function of the temperature for $\boldsymbol{\varepsilon}^{\mathrm{s}}$ (full circles) and for three other nonsaddle point control fields (open circles, triangles, and squares). The ratio $J\left[T, \varepsilon^{\mathrm{s}}\right] / J_{\text {saddle }}(T)$ remains very close to 0.98 , regardless of the temperature. In contrast, for the three other nonsaddle point control fields, the ratio $J[T, \varepsilon] / J_{\text {saddle }}[T]$ changes considerably as the temperature is varied. While $\varepsilon^{\mathrm{s}}(t)$ determined at $T=500 \mathrm{~K}$ identified the saddle at other temperatures, Fig. 2(b) shows that the critical value $J_{\text {saddle }}[T]$ given in Eq. (22) varies significantly with $T$. The results of Figs. 1 and 2 show that the field determined at a critical point will maintain that particular critical character of $J[T]$, irrespective of the temperature.

\section{B. Photoassociation}

Consider a one-dimensional photoassociation model [29,30], where the Hamiltonian in Eq. (17) describes the relative motion of two colliding atoms, and the atoms are on a line aligned with the laser field. The function $V(r)$ is a 
Morse potential with a infinite barrier placed at long range [31], which leads to a nondegenerate energy spectrum composed of $N_{B}$ bound levels $E_{v}^{B}$ with eigenstates $\left|\phi_{v}^{B}\right\rangle$ and $N_{S}$ densely packed discretized scattering levels $E_{n}^{S}$ with eigenstates $\left|\phi_{n}^{S}\right\rangle$. The parameters of the Morse potential in atomic units are $D_{e}=0.0092, r_{e}=0, \alpha=2.1$, and $M=1836$ along with the infinite barrier located at $L=100$ a.u. The dipole function is given by $\mu(r)=q r \exp \left(-r / r_{d}\right)$, where $r_{d}=0.9524$ a.u. and $q=1$ a.u. For the chosen parameters, the number of bound levels is $N_{B}=3$, while the number of scattering levels is truncated to $N_{S}=100$.

The gas corresponding to the model above at thermal equilibrium will contain some fraction of bound diatomic molecules. However, before the control field is turned on, we focus only on the remaining unbound atoms or, equivalently, we consider a nascent gas consisting of atoms at translational thermal equilibrium. Therefore, the initial density operator can be written as

$$
\rho(0, T)=\sum_{n=1}^{N_{S}} \frac{1}{Z} e^{-\beta E_{n}^{S}}\left|\phi_{n}^{S}\right\rangle\left\langle\phi_{n}^{S}\right|,
$$

with $e^{-\beta E_{1}^{S}}>\cdots>e^{-\beta E_{n}^{S}}>\cdots>e^{-\beta E_{N_{S}}^{S}}$ and $Z$ being the partition function associated with the scattering states and thereby satisfying Eq. (14). With the aim of maximizing the overall photoassociation probability, the observable operator is chosen as

$$
O=\sum_{\nu=1}^{N_{B}}\left|\phi_{\nu}^{B}\right\rangle\left\langle\phi_{\nu}^{B}\right|,
$$

where all $N_{B}$ bound vibrational levels are weighted equally. Thus, the goal is to find a field $\varepsilon^{*}(t)$ that maximizes the cost functional $J[T, \varepsilon]=\operatorname{Tr}\left[\rho\left(t_{f}, T\right) O\right]$,

$$
J[T, \boldsymbol{\varepsilon}]=\frac{1}{Z} \sum_{n=1}^{N_{S}} e^{-\beta E_{n}^{S}} P_{n \rightarrow \mathrm{B}},
$$

where

$$
P_{n \rightarrow \mathrm{B}}=\sum_{\nu=1}^{N_{B}}\left|\left\langle\phi_{\nu}^{B}\left|U\left(t_{f}, 0\right)\right| \phi_{n}^{S}\right\rangle\right|^{2}
$$

and $t_{f}=30 \mathrm{ps}$.

The maximum attainable photoassociation yield at a temperature $T$ is

$$
J_{\max }[T]=\frac{1}{Z} \sum_{n=1}^{N_{B}} e^{-\beta E_{n}^{S}} .
$$

This photoassociation upper limit will be reached when the transition probabilities from each one of the lowest $N_{B}$ scattering levels to all of the bound levels are 1, that is, when $P_{n \rightarrow \mathrm{B}}=1, n=1, \ldots, N_{B}$. By the analysis in Sec. II, an optimal control field $\varepsilon^{*}(t)$ determined at a particular temperature $T$ that leads to the maximum limit in Eq. (27) should remain an optimal field independent of the temperature.

At the temperature $T=30 \mathrm{~K}$, we sought an optimized field $\varepsilon^{*}(t)$ starting from a trial field of the form $\boldsymbol{\varepsilon}_{\text {trial }}=\varepsilon_{0} S(t) \sum_{i, j} \sin \left(\omega_{i, j} t\right)$, where $\omega_{i, j}=\left|E_{i}-E_{j}\right| / \hbar$ $(i, j=1, \ldots, N)$ and $S(t)=\sin ^{2}\left(\pi t / t_{f}\right)$. The resultant (nearly
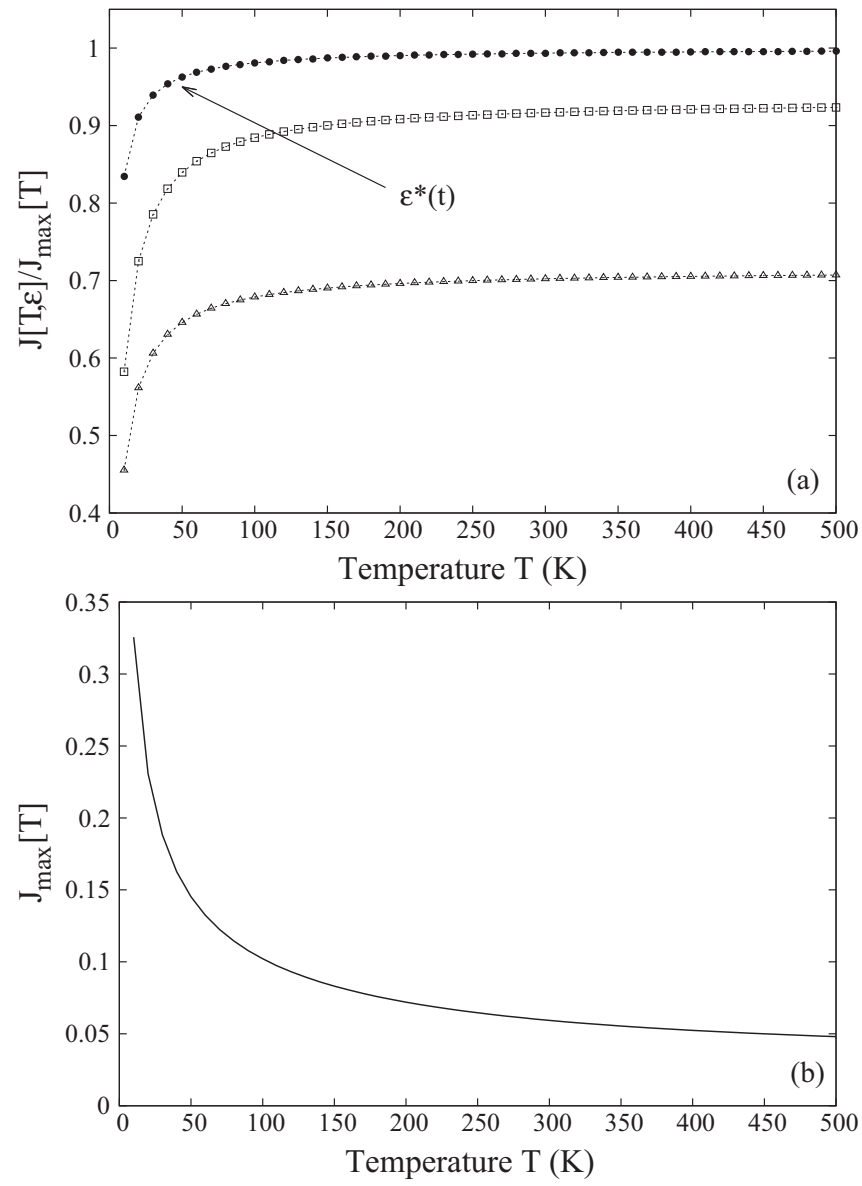

FIG. 3. (a) Ratio $J[T, \varepsilon] / J_{\max }[T]$ as a function of the temperature for photoassociation. Each curve was obtained for a different control field determined during the optimization process performed at $T=30 \mathrm{~K}$. The curve with filled circles corresponds to the control field $\varepsilon^{*}(t)$ producing a yield of $\approx 0.94$ at $T=30 \mathrm{~K}$. All of the fields asymptote to a constant value at high temperature, but only the case of $\varepsilon^{*}(t)$ approaches the optimal limit of 1.0. (b) The value of $J_{\max }[T]$ changes significantly as a function of $T$.

optimal) control field gives the yield $J\left[30 \mathrm{~K}, \boldsymbol{\varepsilon}^{*}\right] /$ $J_{\max }(30 \mathrm{~K}) \approx 0.94$; attaining a higher yield should be possible, but the effort is computationally intensive. Figure 3(a) shows the ratio $J\left[T, \varepsilon^{*}\right] / J_{\max }[T]$ as a function of the temperature $T$ (filled circles) utilizing the field determined at $30 \mathrm{~K}$. Figure 3(b) shows that $J_{\max }[T]$ given in Eq. (27) decreases significantly with the temperature. For comparison, Fig. 3(a) also shows the ratio for two other noncritical control fields (open triangles and squares) which were obtained during the optimization at $T=30 \mathrm{~K}$. For all fields, the ratio increases and approaches a constant value as the temperature increases. This behavior can be understood, since as the temperature increases, $Z J_{\max }[T]=\sum_{n=1}^{N_{B}} e^{-\beta E_{n}^{S}}$ becomes equal to the number of bound states, $N_{B}$, since $\beta E_{n}^{S} \approx 0$. Simultaneously, $Z J[T, \varepsilon]$ also approaches a constant value approximately equal to $\sum_{n=1}^{N} P_{n \rightarrow \mathrm{B}}$, for each control field. Thus, in this model, the ratio between the photoassociation yield for any control and the maximum attainable yield always increases and approaches a constant value as the temperature rises. However, only the case with $\varepsilon^{*}(t)$ produces a ratio that is close to the optimal 
limit 1.0, because for this field the transition probabilities $P_{n \rightarrow \mathrm{B}}$ of the lowest $N_{B}$ scattering levels to all of the bounds are approximately one. Finally, we note that $J[T, \varepsilon] / J_{\max }[T]$ decreases as $T \rightarrow 0$ due to the fact that $J_{\max }[T]$ always increases faster than $J[T, \varepsilon]$ for any nonoptimal field (i.e., $P_{n \rightarrow \mathrm{B}}$ of the lowest $N_{B}$ scattering levels to all of the bound levels are less than 1.0).

\section{CONCLUSION}

This paper presented an analysis of the nature of control fields associated with the critical points of quantum ensemble control problems with respect to experimentally adjustable parameters. We show that a control field $\boldsymbol{\varepsilon}(t)$ that produces a particular critical point on the control landscape will then produce the same critical point independent of the experimental parameters, if the orderings of the eigenvalues of the initial density operator and the target observable operator do not change with respect to these parameters. In particular, when this sufficient condition holds, an optimal control field will remain optimal independent of the experimental parameters. This invariance property can be very important for saving optimization effort in the laboratory and in simulations, as an optimization at one set of parameter values will suffice to produce the same optimal outcome at other values.

\section{ACKNOWLEDGMENTS}

This work was partially supported by the U.S. Department of Energy (grant DE-FG02-02ER15344), National Science Foundation (grant CHE-1058644), and the Army Research Office (grant W911NF-11-1-2068). E.F.L. acknowledges support from UNESP (grant 01/2012 -PROPG) and from National Council for Scientific and Technological Development, CNPqBrazil (grant 473283/2013-1).

\section{APPENDIX A: TBQCP OPTIMIZATION SCEHEM WITHIN THE DENSITY MATRIX FRAMEWORK}

This appendix presents an algorithm to determine an optimal control field within the density matrix framework. The goal is to formulate a monotonically convergent algorithm to find a control field $\boldsymbol{\varepsilon}(t), t \in\left[0, t_{f}\right]$, that can steer the quantum system from an initial state $\rho(0)$ to a final state $\rho\left(t_{f}\right)$ in a finite time $t_{f}$ such that the corresponding expectation value $\left\langle O\left(t_{f}\right)\right\rangle \equiv \operatorname{Tr}\left\{\rho\left(t_{f}\right) O\right\}$ of a given observable operator $O$ is as close as possible to its admissible maximum value. Consider the equation for the density matrix $\rho(t)$ with the Hamiltonian given in Eq. (17):

$$
\frac{\partial}{\partial t} \rho(t)=\frac{1}{\imath \hbar}\left[H_{0}-\mu(r) \boldsymbol{\varepsilon}(t), \rho(t)\right]
$$

In order to extend a recently formulated fast monotonically convergent optimal control search algorithm, the twopoint boundary-value quantum control paradigm (TBQCP) $[26,32,33]$, to mixed-state quantum optimal control problems, we introduce a time-dependent Hermitian operator $\mathcal{O}(t)$ satisfying the equation

$$
\frac{\partial}{\partial t} \mathcal{O}(t)=\frac{1}{\imath \hbar}\left[H_{0}-\mu(r) \boldsymbol{\varepsilon}^{(0)}(t), \mathcal{O}(t)\right],
$$

with the boundary condition $\mathcal{O}\left(t_{f}\right)=O$, associated with a reference control field $\boldsymbol{\varepsilon}^{(0)}(t)$. From Eq. (A2), it can then be shown that the expectation value $\operatorname{Tr}\left\{\rho^{(0)}(t) \mathcal{O}(t)\right\}$ is independent of time $t$, where the density matrix $\rho^{(0)}(t)$ is also associated with the reference control field $\boldsymbol{\varepsilon}^{(0)}(t)$ and satisfies the equation

$$
\frac{\partial}{\partial t} \rho^{(0)}(t)=\frac{1}{l \hbar}\left[H_{0}-\mu(r) \boldsymbol{\varepsilon}^{(0)}(t), \rho^{(0)}(t)\right], \quad \rho^{(0)}(0)=\rho(0),
$$

From Eqs. (A1) and (A2), we may derive the relation

$$
\frac{d}{d t}\langle\mathcal{O}\rangle(t) \equiv \frac{d}{d t} \operatorname{Tr}\{\rho(t) \mathcal{O}(t)\}=f_{\rho}(t)\left\{\boldsymbol{\varepsilon}(t)-\boldsymbol{\varepsilon}^{(0)}(t)\right\},
$$

where

$$
f_{\rho}(t)=-\frac{1}{\imath \hbar} \operatorname{Tr}\{[\mathcal{O}(t), \mu(r)] \rho(t)\} .
$$

Integrating Eq. (A4) leads to

$$
\left\langle\mathcal{O}\left(t_{f}\right)\right\rangle-\langle\mathcal{O}(0)\rangle=\int_{0}^{t_{f}} f_{\rho}(t)\left\{\boldsymbol{\varepsilon}(t)-\boldsymbol{\varepsilon}^{(0)}(t)\right\} d t
$$

which is a nonlinear integral equation for the control field $\boldsymbol{\varepsilon}(t)$. From Eqs. (A4) and (A6), a monotonically convergent iteration scheme can be obtained by choosing the control field $\boldsymbol{\varepsilon}(t)$ according to the relation $[26,32,33]$

$$
\boldsymbol{\varepsilon}(t)=\boldsymbol{\varepsilon}^{(0)}(t)+\eta_{0} S(t) f_{\rho}(t),
$$

where $\eta_{0}>0$, and $S(t)$ is a non-negative envelope function for the control field. Substituting Eq. (A7) into Eq. (A6) gives

$$
\left\langle\mathcal{O}\left(t_{f}\right)\right\rangle-\langle\mathcal{O}(0)\rangle=\eta \int_{0}^{t_{f}} S(t) f_{\rho}^{2}(t) d t \geqslant 0,
$$

i.e., $\left\langle\mathcal{O}\left(t_{f}\right)\right\rangle=\left\langle O\left(t_{f}\right)\right\rangle=\operatorname{Tr}\left\{\rho\left(t_{f}\right) O\right\} \geqslant \operatorname{Tr}\left\{\rho^{(0)}\left(t_{f}\right) O\right\}=\langle\mathcal{O}(0)\rangle$. Equation (A7) forms a recurrence relation for the two-point boundary-value quantum optimal paradigm for the density matrix that can be applied iteratively to refine the control field such that the targeted expectation $\left\langle O\left(t_{f}\right)\right\rangle$ increases monotonically.

\section{APPENDIX B: FINDING KINEMATIC CRITICAL POINTS}

This appendix shows how a field may be found which corresponds to a specific suboptimal critical point of the control landscape $J[\varepsilon]$. We use the same definitions of Sec. II, but without explicitly labeling with the parameters $X$ and $Y$.

Consider a new cost functional

$$
\mathcal{J}\left[\boldsymbol{\varepsilon}_{\Pi}\right] \equiv \operatorname{Tr}\left\{\rho^{\Pi}(T) O\right\}=\operatorname{Tr}\left\{U_{\Pi}(T, 0) \rho^{\Pi}(0) U_{\Pi}^{\dagger}(T, 0) O\right\}
$$

associated with the observable $O$ and with a modified initial density matrix

$$
\rho^{\Pi}(0)=\Pi \rho(0) \Pi^{T},
$$

where $\Pi$ is a permutation matrix and $U_{\Pi}(t, 0)$ is the evolution operator associated with a control field $\boldsymbol{\varepsilon}_{\Pi}(t), t \in\left[0, t_{f}\right]$. Thus, the new initial density matrix $\rho^{\Pi}(0)$ and the initial density matrix $\rho(0)$ contain the same set of eigenvalues, 
but with a different ordering. Furthermore, Eq. (B1) can be written as

$$
\begin{aligned}
\mathcal{J}\left[\varepsilon_{\Pi}\right] & =\operatorname{Tr}\left\{\rho(0) U^{\prime \dagger}(T, 0) D^{\prime} \Lambda^{\prime} D^{\prime \dagger} U^{\prime}(T, 0)\right\} \\
& =\operatorname{Tr}\left\{\rho(0) U^{\prime \dagger}(T, 0) O^{\prime} U^{\prime}(T, 0)\right\},
\end{aligned}
$$

where

$$
\begin{aligned}
U^{\prime}(T, 0) & =\Pi^{T} U_{\Pi}(T, 0) \Pi, \\
D^{\prime} & =\Pi^{T} D \Pi, \\
\Lambda^{\prime} & =\Pi^{T} \Lambda \Pi,
\end{aligned}
$$

and

$$
O^{\prime}=D^{\prime} \Lambda^{\prime} D^{\prime \dagger}=\Pi^{T} O \Pi,
$$

with $U^{\prime}(t, 0)$ being the evolution operator associated with a control field $\boldsymbol{\varepsilon}_{\Pi}(t)$ and the transformed Hamiltonian $H^{\prime}(t)=$ $\Pi^{T} H(t) \Pi$. Here $O^{\prime}$ and $O$ contain the same set of eigenvalues, but with permuted ordering, according to Eq. (B6).

It is easily seen that a critical control $\varepsilon_{\Pi}(t)$ on the landscape $\mathcal{J}\left[\varepsilon_{\Pi}\right]$ satisfies the commutation relation

$$
\left[\rho_{\text {critical }}^{\Pi}\left(t_{f}\right), O\right]=0,
$$

or equivalently,

$$
\left[\rho^{\Pi}(0), O_{\text {critical }}^{\Pi}\left(t_{f}\right)\right]=0,
$$

where

$$
O^{\Pi}\left(t_{f}\right) \equiv U_{\Pi}^{\dagger}\left(t_{f}, 0\right) O U_{\Pi}\left(t_{f}, 0\right)
$$

and

$$
\rho^{\Pi}\left(t_{f}\right)=U_{\Pi}\left(t_{f}, 0\right) \rho^{\Pi}(0) U_{\Pi}^{\dagger}\left(t_{f}, 0\right) .
$$

Assuming that $\rho(0)$ is fully nondegenerate, Eq. (B9) leads to the relations

$$
\mathcal{J}_{\text {critical }}\left[\varepsilon_{\Pi}\right]=\operatorname{Tr}\left\{\rho^{\Pi}(0) \Pi^{\prime T} \Lambda \Pi^{\prime}\right\},
$$

where $\Pi^{\prime}$ is an arbitrary permutation matrix. Comparing Eq. (12) with the above expression, we conclude that every kinematic critical point of $\mathcal{J}\left[\varepsilon_{\Pi}\right]$ is also a kinematic critical point of $J[\varepsilon]$. In particular, both control landscapes have the same maximum,

$$
\mathcal{J}_{\max }\left[\varepsilon_{\Pi}\right]=\mathcal{J}\left[\boldsymbol{\varepsilon}_{\Pi}^{\mathrm{opt}}\right]=\sum_{j=1}^{N} p_{j} \lambda_{j}=J_{\max }[\varepsilon],
$$

where $\varepsilon_{\Pi}^{\text {opt }}$ is an optimal control field that maximizes $\mathcal{J}\left[\boldsymbol{\varepsilon}_{\Pi}\right]$. Finally, if $J[\varepsilon]$ is evaluated at $\varepsilon_{\Pi}^{\text {opt }}$, we obtain

$$
\begin{aligned}
J\left[\varepsilon_{\Pi}^{\mathrm{opt}}\right] & =\operatorname{Tr}\left\{U^{\prime}(T, 0) \rho(0) U^{\prime \dagger}(T, 0) O\right\} \\
& =\operatorname{Tr}\left\{\Pi^{T} D \rho(0) D^{\dagger} \Pi O\right\} \\
& =\operatorname{Tr}\left\{\rho(0) D^{\dagger} \Pi D \Lambda D^{\dagger} \Pi^{T} D\right\} \\
& \leqslant \operatorname{Tr}\{\rho(0) \Lambda\}=J_{\max }[\varepsilon] .
\end{aligned}
$$

Therefore, $\varepsilon_{\Pi}^{\mathrm{opt}}(t)$ corresponds to a saddle of $J[\varepsilon]$. All of the kinematic critical points of the two control landscapes $J[\varepsilon]$ and $\mathcal{J}\left[\varepsilon_{\Pi}\right]$ are equivalent through a permutation. As a result, we can search for kinematic critical points using the same algorithm of Appendix A.
[1] S. A. Rice and M. Zhao, Optimal Control of Molecular Dynamics (Wiley-Interscience, New York, 2000).

[2] H. Rabitz, R. Vivie-Riedle, M. Motzkus, and K. Kompa, Science 288, 824 (2000).

[3] J. Werschnik and E. K. U. Gross, J. Phys. B 40, R175 (2007).

[4] C. Brif, R. Chakrabarti, and H. Rabitz, New J. Phys. 12, 075008 (2010).

[5] P. v. d. Hoff, S. Thallmair, M. Kowalewski, R. Siemering, and R. d. Vivie-Riedle, Phys. Chem. Chem. Phys. 14, 14460 (2012).

[6] F. Caruso, S. Montangero, T. Calarco, S. F. Huelga, and M. B. Plenio, Phys. Rev. A 85, 042331 (2012).

[7] E. Räsänen and L. B. Madsen, Phys. Rev. A 86, 033426 (2012).

[8] E. Räsänen and E. Heller, Eur. Phys. J. B 86, 1 (2013).

[9] G. Jäger and U. Hohenester, Phys. Rev. A 88, 035601 (2013).

[10] M. Hellgren, E. Räsänen, and E. K. U. Gross, Phys. Rev. A 88, 013414 (2013).

[11] T.-S. Ho, J. Dominy, and H. Rabitz, Phys. Rev. A 79, 013422 (2009).

[12] G. M. Huang, T. J. Tarn, and J. W. Clark, J. Math. Phys. 24, 2608 (1983)

[13] V. Ramakrishna, M. V. Salapaka, M. Dahleh, H. Rabitz, and A. Peirce, Phys. Rev. A 51, 960 (1995).
[14] S. G. Schirmer, H. Fu, and A. I. Solomon, Phys. Rev. A 63, 063410 (2001).

[15] R.-B. Wu, R. Long, J. Dominy, T.-S. Ho, and H. Rabitz, Phys. Rev. A 86, 013405 (2012).

[16] K. W. Moore and H. Rabitz, Phys. Rev. A 84, 012109 (2011).

[17] H. Rabitz, T.-S. Ho, R. Long, R. Wu, and C. Brif, Phys. Rev. Lett. 108, 198901 (2012).

[18] O. W. Sørensen, J. Magn. Reson. (1969) 86, 435 (1990).

[19] J. Stoustrup, O. Schedletzky, S. J. Glaser, C. Griesinger, N. C. Nielsen, and O. W. Sørensen, Phys. Rev. Lett. 74, 2921 (1995).

[20] M. D. Girardeau, S. G. Schirmer, J. V. Leahy, and R. M. Koch, Phys. Rev. A 58, 2684 (1998).

[21] T. S. Untidt and N. C. Nielsen, J. Chem. Phys. 113, 8464 (2000).

[22] H. A. Rabitz, M. M. Hsieh, and C. M. Rosenthal, Science 303, 1998 (2004).

[23] T.-S. Ho and H. Rabitz, J. Photochem. Photobiol. A 180, 226 (2006).

[24] R. Chakrabarti and H. Rabitz, Int. Rev. Phys. Chem. 26, 671 (2007).

[25] T.-S. Ho, H. Rabitz, and G. Turinici, Acta Appl. Math. 118, 49 (2012).

[26] T.-S. Ho and H. Rabitz, Phys. Rev. E 82, 026703 (2010).

[27] H. Guo, J. Chem. Phys. 99, 1685 (1993). 
[28] P. Marquetand, M. Richter, J. Gonzalez-Vazquez, I. Sola, and L. Gonzalez, Faraday Discuss. 153, 261 (2011).

[29] E. F. de Lima, T.-S. Ho, and H. Rabitz, Phys. Rev. A 78, 063417 (2008).

[30] E. F. de Lima, T.-S. Ho, and H. Rabitz, Chem. Phys. Lett. 501, 267 (2011)
[31] E. F. de Lima, T.-S. Ho, and H. Rabitz, J. Phys. A 41, 335303 (2008).

[32] T.-S. Ho, H. Rabitz, and S.-I. Chu, Comput. Phys. Commun. 182, 14 (2011).

[33] S.-L. Liao, T.-S. Ho, S.-I. Chu, and H. Rabitz, Phys. Rev. A 84, 031401(R) (2011). 